\title{
Finite temperature fluctuation-induced order and responses in magnetic topological insulators
}

\author{
Marius Scholten $\odot,{ }^{1}$ Jorge I. Facio, ${ }^{1}$ Rajyavardhan Ray $\odot,{ }^{1,2}$ Ilya M. Eremin $\odot,{ }^{3}$ \\ Jeroen van den Brink, ${ }^{1,4}$ and Flavio S. Nogueira $\circledast^{1}$ \\ ${ }^{1}$ Institute for Theoretical Solid State Physics, IFW Dresden, Helmholtzstrasse 20, D-01069 Dresden, Germany \\ ${ }^{2}$ Dresden Center for Computational Materials Science (DCMS), TU Dresden, D-01062 Dresden, Germany \\ ${ }^{3}$ Institut für Theoretische Physik III, Ruhr-Universität Bochum, D-44780 Bochum, Germany \\ ${ }^{4}$ Institute for Theoretical Physics and Würzburg-Dresden Cluster of Excellence ct.qmat, TU Dresden, D-01069 Dresden, Germany
}

(Received 21 January 2021; accepted 22 June 2021; published 9 July 2021)

\begin{abstract}
We derive an effective field theory model for magnetic topological insulators and predict that a magnetic electronic gap persists on the surface for temperatures above the ordering temperature of the bulk. Our analysis also applies to interfaces of heterostructures consisting of a ferromagnetic and a topological insulator. In order to make quantitative predictions for $\mathrm{MnBi}_{2} \mathrm{Te}_{4}$ and for $\mathrm{EuS}-\mathrm{Bi}_{2} \mathrm{Se}_{3}$ heterostructures, we combine the effective field theory method with density functional theory and Monte Carlo simulations. For $\mathrm{MnBi}_{2} \mathrm{Te}_{4}$ we predict an upwards Néel temperature shift at the surface up to $15 \%$, while the EuS- $\mathrm{Bi}_{2} \mathrm{Se}_{3}$ interface exhibits a smaller relative shift. The effective theory also predicts induced Dzyaloshinskii-Moriya interactions and a topological magnetoelectric effect, both of which feature a finite temperature and chemical potential dependence.
\end{abstract}

DOI: 10.1103/PhysRevResearch.3.L032014

Introduction. Since the first experimental observation of the quantum anomalous Hall effect (QAHE) — the appearance of quantized Hall conductance at zero magnetic field-in thin films of the topological insulator (TI) $\mathrm{Bi}_{2} \mathrm{Se}_{3}$ doped with magnetic atoms at temperatures below $1 \mathrm{~K}$ [1], magnetic topological materials have been at the scientific forefront both experimentally and theoretically [2-11]. The QAHE requires a three-dimensional TI in which long-range magnetic order breaks the time-reversal symmetry, via ferromagnetic [12-15] or antiferromagnetic ordering [16]. Driven by the major goal to realize quantization of conductance at room temperature, two distinct directions of material development have triggered much of the recent experimental progress: the successful fabrication of atomically sharp interfaces of ferromagnetic and topological materials, in particular of EuS and $\mathrm{Bi}_{2} \mathrm{Se}_{3}$ [12-14], and the growth of intrinsically magnetic TIs such as $\operatorname{MnTe}\left(\mathrm{Bi}_{2} \mathrm{Te}_{3}\right)_{m}$ with $m \geqslant 1$ [17-22] and $\mathrm{MnSb}_{2} \mathrm{Te}_{4}[23,24]$ as highly ordered single crystals or their intrinsic heterostructures $[25,26]$.

Conceptually, a perfectly quantized Hall conductivity of $e^{2} / h$ arises at zero temperature when the magnetization couples to the topological Dirac-type TI surface states, opening up a gap there in which then the chemical potential $\mu$ must lie (see Fig. 1). As a bonus, fermionic quantum fluctuations induce a concomitant linear topological magnetoelectric effect (TME), which couples electric fields directly to the magnetization and, vice versa, magnetization dynamics to

Published by the American Physical Society under the terms of the Creative Commons Attribution 4.0 International license. Further distribution of this work must maintain attribution to the author(s) and the published article's title, journal citation, and DOI. electrical polarization [27,28]. At zero temperature for $\mu$ outside the gap both Hall conductance and TME fail to be quantized $[2,6,29]$, tending to vanish as $\mu$ grows. Nevertheless, this particular situation has the interesting feature, that a Dzyaloshinskii-Moriya interaction (DMI) between magnetic degrees of freedom emerges, opening the path towards the formation of various skyrmionlike topological magnetic textures at the surface [30,31], observed also experimentally [32].

The goal to realize the QAHE, TME, and possibly even a DMI at room temperature ties into a number of fundamental and practical questions. The first is how in an ideal situation the surface magnetic ordering temperature $T_{\mathrm{c}}$ is affected by coupling to the topological edge states - it has been suggested that this coupling can greatly enhance $T_{\mathrm{c}}$ [12,33]. Subsequently the question is how temperature fluctuations affect the conductance quantization, TME, and DMI for the different relevant regimes of $\mu$. Particularly interesting would be the existence of a temperature regime in which both DMI and TME are sizable, in which case the TME endows external magnetic and electric fields with novel types of access to DMI-induced skyrmions.

Despite several theoretical and experimental developments in recent years, a number of fundamental questions remain to be answered. For instance, although a remarkable enhancement of $T_{\mathrm{c}}$ at the interfaces of certain ferromagnetic insulators (FMIs) have been reported [12,34], recent works [35,36] question the validity of these findings for the specific case of EuS proximate to either $\mathrm{Bi}_{2} \mathrm{Se}_{3}$ or $(\mathrm{Bi}, \mathrm{Sb})_{2} \mathrm{Te}_{3}$. Furthermore, a recent experimental work [35] indicates that the topological electronic states at the interface do not interact strongly with ferromagnetism for the case of a $\mathrm{EuS}-\mathrm{Bi}_{2} \mathrm{Se}_{3}$ heterostructure. Additionally, in the family of magnetic TIs $\mathrm{MnTe}\left(\mathrm{Bi}_{2} \mathrm{Te}_{3}\right)_{m}$, different works that do find a surface spectrum gapped below the Néel temperature also observe the persistence of the gap 
in the paramagnetic phase $[17,22,37]$, it being unclear whether or not these observations result from the intrinsic magnetism.

Here, we develop the finite temperature continuum field theory to address the questions concerning the magnetic phase transitions and dynamics at finite temperature, and apply these results to several experimentally relevant material systems, using Monte Carlo simulations (MCSs) and density functional theory (DFT) based approaches to obtain quantitative results. Using a minimal model for the coupling of the Dirac fermions to the magnetic Hamiltonian, we show that in a temperature window where bulk magnetism is absent, an out-of-plane surface magnetization can still be nonzero and induce a gap in the Dirac spectrum. As a consequence, the AHE and TME can survive in a certain temperature range above the bulk $T_{\mathrm{c}}$. However, for the experimentally relevant materials and material combinations (EuS- $\mathrm{Bi}_{2} \mathrm{Se}_{3}, \mathrm{MnBi}_{2} \mathrm{Te}_{4}$ ), the coupling to the topological surface states enhances the bulk $T_{\mathrm{c}}$ not more than $15 \%$, even under the most favorable conditions. Significant enhancements would require using TIs with a much lower Fermi velocity of the Dirac cone. In addition, at finite $\mu$ we establish the existence of a temperature regime displaying both a substantial fluctuation-induced DMI and TME, even if its Hall conductivity is strongly renormalized, with potentially interesting consequences for skyrmion manipulation and transport.

Saddle-point and induced order. To determine the shift in magnetic ordering temperature due to coupling of the magnetic moments to the fluctuating Dirac fermions, we consider the following minimal model Hamiltonian,

$$
H_{\text {Dirac }}=\left[\hbar v_{\mathrm{F}} \boldsymbol{d}(-i \nabla)-J_{0} \boldsymbol{n}(\boldsymbol{r}, t)\right] \cdot \sigma-e \phi(\boldsymbol{r}, t)-\mu,
$$

where the Dirac fermions couple to the magnetization via a magnetic exchange interaction $J_{0}, \sigma$ is the Pauli matrix vector, and $\boldsymbol{n}(\boldsymbol{r}, t)$ the unit vector field representing the magnetization direction at $\boldsymbol{r}=(x, y)$. The operator $\boldsymbol{d}(-i \nabla)$ has the property $\boldsymbol{d}^{2}=-\nabla^{2}$, where $\nabla=\left(\partial_{x}, \partial_{y}, 0\right)$. Additionally, an electric potential $\phi$ has been introduced, which includes contributions of an externally applied electric field and an internal longrange Coulomb interaction as well.

The fermionic quantum fluctuations of the Dirac Hamiltonian (1) are accounted for by the imaginary time path integral,

$$
\begin{aligned}
& Z_{\mathrm{F}}=e^{-\beta \mathcal{F}_{\mathrm{F}}(\boldsymbol{n})}=\int \mathcal{D}\left[\Psi^{\dagger}, \Psi\right] e^{-\frac{1}{\hbar} S\left[\Psi^{\dagger}, \Psi\right]}, \\
& S=\int_{0}^{\hbar \beta} d \tau \int d^{2} r \Psi^{\dagger}\left(\hbar \partial_{\tau}+H_{\text {Dirac }}\right) \Psi,
\end{aligned}
$$

where $\Psi=\left(\Psi_{\uparrow}, \Psi_{\downarrow}\right)^{T}$ is a spinor of Grassmann fields obtained from the second-quantized Hamiltonian [38]. The above partition function defines a free-energy functional $\mathcal{F}_{\mathrm{F}}(\boldsymbol{n})$ which provides an additional free energy to the one of the magnetic free energy. As a minimal model leading to the latter, we consider the magnetic Hamiltonian,

$$
H_{\mathrm{M}}=\int d^{2} r\left[\frac{J}{2}(\nabla \boldsymbol{n})^{2}-\frac{K}{2} n_{z}^{2}\right],
$$

where $J>0$ is the exchange energy and $K>0$ is the anisotropy energy density (per unit area). The magnetic partition function is given by the path integral,

$$
z_{\mathrm{M}}=\int \mathcal{D} \boldsymbol{n} \mathcal{D} \lambda e^{-\frac{1}{\hbar} S_{\mathrm{B}}-\frac{1}{\hbar} \int_{0}^{\hbar \beta} d \tau\left[H_{\mathrm{M}}+\frac{i}{2} \int d^{2} r \lambda\left(\boldsymbol{n}^{2}-1\right)\right]},
$$

where $S_{\mathrm{B}}$ is the Berry phase that arises in the construction of the spin coherent state path integral [39], and $\lambda$ is a Lagrange multiplier field enforcing the constraint $\boldsymbol{n}^{2}=1$.

Keeping the magnetic fluctuations classical, we obtain the following effective Hamiltonian after integrating out the Gaussian fluctuations $n_{x}$ and $n_{y}$, along with the fermions,

$$
\begin{aligned}
H_{\mathrm{eff}}= & k_{\mathrm{B}} T \operatorname{Tr} \ln \left(-J \nabla^{2}+i \lambda\right) \\
& -k_{\mathrm{B}} T \operatorname{Tr} \ln \left[\hbar \partial_{\tau}-\mu+\hbar v_{\mathrm{F}} \boldsymbol{d}(-i \nabla) \cdot \sigma-J_{0} n_{z} \sigma_{z}\right] \\
& +\frac{1}{2} \int d^{2} r\left[J\left(\nabla n_{z}\right)^{2}-K n_{z}^{2}+i \lambda\left(n_{z}^{2}-1\right)\right] .
\end{aligned}
$$

Variation with respect to $n_{z}$ leads to the saddle-point equation,

$$
\left(\lambda_{0}-K\right) n_{z}=2 J_{0}^{2} n_{z} k_{\mathrm{B}} T \sum_{n} \int \frac{d^{2} q}{(2 \pi)^{2}} \frac{1}{\left(\hbar \omega_{n}+i \mu\right)^{2}+E_{q}^{2}},
$$

where $E_{q}=\sqrt{\left(\hbar v_{\mathrm{F}} q\right)^{2}+m^{2}}, \quad \omega_{n}=\pi k_{\mathrm{B}} T(2 n+1) / \hbar$ is a fermionic Matsubara frequency, and we have defined $m^{2}=$ $J_{0}^{2} n_{z}^{2}$. Equation (7) is solved together with the saddle-point equation for $\lambda$, which occurs at $i \lambda=\lambda_{0}$,

$$
n_{z}^{2}=1-\frac{2 k_{\mathrm{B}} T}{J} \int \frac{d^{2} q}{(2 \pi)^{2}} \frac{1}{q^{2}+\lambda_{0} / J} .
$$

Setting $J_{0}=0$ in Eq. (7) reduces the saddle-point equations to one of a classical ferromagnet with easy-axis anisotropy. In this special case the ordered phase immediately implies $\lambda_{0}=$ $K$ and from Eq. (8) it is straightforward to obtain the critical temperature $T_{\mathrm{c}}$ by demanding that $n_{z}\left(T_{\mathrm{c}}\right)=0$, yielding

$$
k_{\mathrm{B}} T_{\mathrm{c}}=\frac{\pi J}{\ln \left(\Lambda_{\mathrm{s}} \sqrt{\frac{J}{K}}\right)},
$$

where a cutoff $\Lambda_{\mathrm{s}} \gg \sqrt{K / J}$ has been introduced. Note that the above is consistent with the Mermin-Wagner theorem in the limit $K \rightarrow 0$.

Our aim is to calculate the shift of this critical temperature when $J_{0} \neq 0$, i.e., accounting for the fermionic quantum fluctuations. After explicitly evaluating the Matsubara sum and integral, Eq. (7) becomes

$$
\begin{aligned}
\lambda_{0}= & -\frac{J_{0}^{2} k_{\mathrm{B}} T}{2 \pi\left(\hbar v_{\mathrm{F}}\right)^{2}}\left[\ln \left(1+e^{-\frac{|m|-\mu}{k_{\mathrm{B}} T}}\right)+\ln \left(1+e^{-\frac{|m|+\mu}{k_{\mathrm{B}} T}}\right)\right] \\
& +K+\frac{J_{0}^{2} \Lambda_{\mathrm{F}}}{2 \pi \hbar v_{\mathrm{F}}}
\end{aligned}
$$

where the cutoff $\Lambda_{\mathrm{F}} \gg|m| /\left(\hbar v_{\mathrm{F}}\right)$. The chemical potential is temperature dependent and can be obtained by fixing the particle density. At the critical temperature $T=\widetilde{T}_{\mathrm{c}}$ of the interacting system we demand that $m=0$ and obtain

$$
\lambda_{0 \mathrm{c}}=K+\frac{J_{0}^{2} \Lambda_{\mathrm{F}}}{2 \pi \hbar v_{\mathrm{F}}}-\frac{J_{0}^{2} k_{\mathrm{B}} \widetilde{T}_{\mathrm{c}}}{2 \pi\left(\hbar v_{\mathrm{F}}\right)^{2}} \ln \left[2+2 \cosh \left(\mu_{\mathrm{c}} / k_{\mathrm{B}} \widetilde{T}_{\mathrm{c}}\right)\right],
$$


where $\mu_{\mathrm{c}}=\mu\left(\widetilde{T}_{\mathrm{c}}\right)$. This finally yields the critical temperature shift relative to the situation where fermions are absent,

$$
\frac{T_{\mathrm{c}}-\widetilde{T}_{\mathrm{c}}}{\widetilde{T}_{\mathrm{c}}}=\frac{k_{\mathrm{B}} T_{\mathrm{c}}}{2 \pi J} \ln \left(\frac{K}{\lambda_{0 \mathrm{c}}}\right) .
$$

Since the cutoff is large, it is clear that the argument of the logarithm in Eq. (12) is smaller than unity, and therefore $\widetilde{T}_{\mathrm{c}}>$ $T_{\mathrm{c}}$ in all cases. From Eqs. (11) and (12) we see that smaller values of $v_{F}$ favor larger shifts of the critical temperature.

Material specifics. In order to make quantitative predictions for the material systems of interest, we need to determine the values of the coupling parameters and cutoffs appearing in the continuum theory. We base such values on ab initio and Monte Carlo calculations, which we find to be consistent with available experimental data.

Based on the DFT for a finite slab, for $\mathrm{MnBi}_{2} \mathrm{Te}_{4}$ we find the Fermi velocity as $\hbar v_{\mathrm{F}}=2.3 \pm 0.2 \mathrm{eV} \AA$ and the coupling $J_{0} \approx 50 \mathrm{meV}$, whereas for $\mathrm{EuS}-\mathrm{Bi}_{2} \mathrm{Se}_{3}$, we consider $\hbar v_{\mathrm{F}} \approx 3.3 \mathrm{eV} \AA$ and $J_{0} \approx 54 \mathrm{meV}[13,40,41]$. Note, the value of $J_{0}$ was derived from the gap size in the Dirac dispersion assuming its origin is purely magnetic. In reality, higherorder effects such as the hybridization of the surface fermions with the bulk electronic states of $\mathrm{MnBi}_{2} \mathrm{Te}_{4}$ or EuS may also influence the gap such that the actual magnetic gap and correspondingly $J_{0}$ might be smaller.

For the fermionic cutoff $\Lambda_{\mathrm{F}}$, we consider that the average surface density of a completely filled band is $1 / A$, with $A$ the surface unit cell area. Since our model describes two surface bands, we fix $\Lambda_{\mathrm{F}}$ such that $n(\mu=0)=1 / A$. Electron-hole symmetry of the model then implies that $n(\mu \rightarrow \infty)=2 / A$.

To set the anisotropy $K$ and stiffness $J$ of the magnetization field, we first build an anisotropic Heisenberg lattice model which we then map to Eq. (4). As the on-site anisotropy depends crucially on the thickness of the FMI layer in the $\mathrm{EuS}-\mathrm{Bi}_{2} \mathrm{Se}_{3}$ system [12], while the Mn layers in $\mathrm{MnBi}_{2} \mathrm{Te}_{4}$ are well separated with relatively small out-ofplane exchange couplings $[17,42]$, we consider the magnetic subsystem to be monolayer thick. The corresponding lattice in both cases is a two-dimensional triangular lattice spanned by $\mathrm{Mn}$ in $\mathrm{MnBi}_{2} \mathrm{Te}_{4}$ and $\mathrm{Eu}$ on the $\mathrm{EuS}(111)$ surface [12]. The considered magnetic interactions comprise nearest-neighbor ferromagnetic exchange couplings $\mathcal{J}$, and an effective on-site out-of-plane anisotropy $\mathcal{K} . J$ and $K$ follow from $\mathcal{J}$ and $\mathcal{K}$ taking the continuum limit [43].

For $\mathrm{MnBi}_{2} \mathrm{Te}_{4}$, we obtain from DFT calculations $\mathcal{K} \approx$ $0.073 \mathrm{meV}$, and $\mathcal{J} \approx 0.18 \mathrm{meV}$, in good agreement with earlier reported values [44]. For monolayer EuS, on the other hand, $\mathcal{K}$ was obtained by extrapolating the data for the 20 -nm-thick $\mathrm{Bi}_{2} \mathrm{Se}_{3}$ layer in the EuS- $\mathrm{Bi}_{2} \mathrm{Se}_{3}$ heterostructures in Ref. [12] to the EuS monolayer thickness. We obtain $\mathcal{K} \approx 0.13 \mathrm{meV}$. The exchange coupling for the monolayer has been previously estimated to be $\mathcal{J}=0.017 \mathrm{meV}$ [45]. It is interesting that these two material systems cover a broad range of $\mathcal{K} / \mathcal{J}$ (from $\sim 0.4$ in the Mn-based compound to 7.6 in the EuS-based system).

Last, the spin cutoff was fixed such that the critical temperature of the continuum model (without fermions) matches the critical temperature of the corresponding Heisenberg model. To obtain the latter, classical MCS for the lattice model were carried out [see the Supplemental Material (SM) [43] for
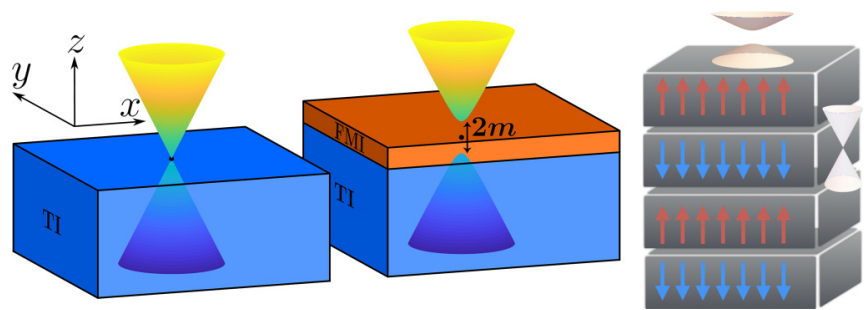

FIG. 1. Left: Symmetry breaking induced by the proximity effect. An exchange coupling is induced across the interface between a FMI and a TI. The FMI polarizes the TI surface by the proximity effect and gaps the surface spectrum as, e.g., $\mathrm{EuS}-\mathrm{Bi}_{2} \mathrm{Se}_{3}$ heterostructures [13]. Right: Intrinsic spontaneous symmetry breaking. Here, the $\mathrm{TI}$ is itself a magnetic insulator such as, e.g., $\mathrm{MnBi}_{2} \mathrm{Te}_{4}$ [17].

details]. For the EuS- $\mathrm{Bi}_{2} \mathrm{Se}_{3}$ and $\mathrm{MnBi}_{2} \mathrm{Te}_{4}$ system, we obtain $T_{\mathrm{c}}^{\text {latt }} \approx 5.8 \mathrm{~K}$ and $\approx 17.0 \mathrm{~K}$, respectively. Based on these values, we fix $\Lambda_{\mathrm{s}}$ via Eq. (9).

Finally, using Eq. (12), we obtain $\left(T_{\mathrm{c}}-\widetilde{T}_{\mathrm{c}}\right) / \widetilde{T}_{\mathrm{c}}=10.9 \%$ and $14.7 \%$ for $\mathrm{EuS}_{-} \mathrm{Bi}_{2} \mathrm{Se}_{3}$ and $\mathrm{MnBi}_{2} \mathrm{Te}_{4}$, respectively. Note that the compound having a smaller Fermi velocity $\left(\mathrm{MnBi}_{2} \mathrm{Te}_{4}\right)$ shows indeed a larger shift of the critical temperature (recall that in both cases $J_{0}$ is similar).

Fluctuations around the saddle point. The question on how the DMI, TME, and Hall conductivity evolve with temperature and chemical potential requires considering the effect of magnetic fluctuations in the fermionic determinant resulting from integrating out the fermions. Introducing $\boldsymbol{n}(\boldsymbol{r}, t)=$ $n_{z} \hat{\mathbf{z}}+\delta \boldsymbol{n}(\boldsymbol{r}, t)$ we can determine the effective action around the saddle-point approximation up to quadratic order in the fluctuations.

The reason for the appearance of the DMI term [30] is that the magnetization fluctuations effectively break the inversion symmetry of our starting Hamiltonian. As a consequence, this yields a DMI contribution to the magnetic free energy,

$$
F_{\mathrm{DMI}}=i \frac{D}{2} \int d^{2} r \delta \boldsymbol{n} \cdot[\boldsymbol{d}(-i \nabla) \times \delta \boldsymbol{n}],
$$

where

$$
D=-\frac{J_{0}^{2}}{4 \pi \hbar v_{\mathrm{F}}} \frac{\sinh (\beta \mu)}{\cosh (\beta \mu)+\cosh (\beta m)} .
$$

It is important to emphasize that the DMI term is not introduced in an ad hoc way-it is generated by charge fluctuations coupling to the magnetic moments at the interface. The DMI vanishes at the neutrality point and is nonzero away from it. This creates the possibility of manipulating the DMI by controlling the chemical potential, for instance, by gating. If we take the mass $m$ to have a mean-field-like behavior $m(T)=J_{0} \sqrt{1-T / \widetilde{T}_{\mathrm{c}}}$, we find the zero temperature value for the DMI $D(T \rightarrow 0)=-\frac{J_{0}^{2}}{4 \pi \hbar v_{\mathrm{F}}} \operatorname{sgn}\left(\epsilon_{\mathrm{F}}\right) H\left(\left|\epsilon_{\mathrm{F}}\right|-\left|J_{0}\right|\right)$ which demonstrates that the DMI kicks in when the Fermi energy $\epsilon_{\mathrm{F}}$ surpasses a threshold given by the exchange coupling constant $J_{0}$. This feature of the generated DMI can also be seen in Fig. 2(a) where we show the whole temperature range for different values of the chemical potential with an estimated zero temperature exchange coupling constant of $J_{0} / k_{\mathrm{B}} \widetilde{T}_{\mathrm{c}} \approx 29.1$ based on our findings for the $\mathrm{MnBi}_{2} \mathrm{Te}_{4}$ system. We further see that the lower the temperature gets, the narrower the range for 

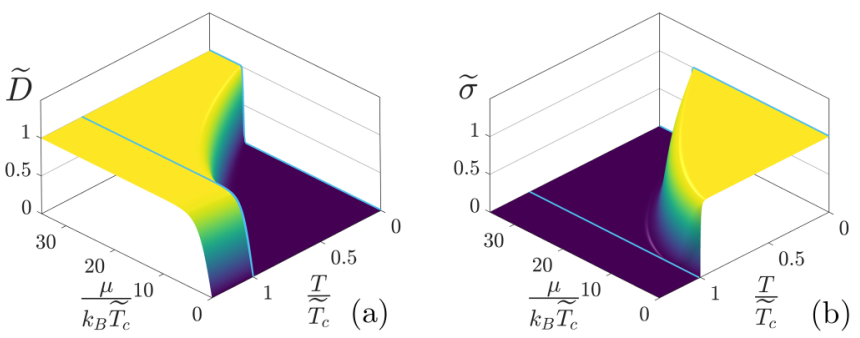

FIG. 2. (a) Normalized massive DMI coupling strength $\tilde{D}=$ $D / \bar{D}$ and (b) normalized topological mass $\tilde{\sigma}=\sigma / \bar{\sigma}$, both as a function of temperature and chemical potential. The normalizations are set to $\bar{D}=-J_{0}^{2} / 4 \pi \hbar v_{\mathrm{F}}$ and $\bar{\sigma}=e^{2} / 2 h$, respectively. The DMI is only present when the chemical potential exceeds the gap (metallic regime). On the contrary the topological mass only exists for a chemical potential inside the gap (insulating regime).

the chemical potential becomes in which there still is a finite DMI. Moreover, we see that the step-function behavior of the generated DMI at zero temperature approximately extends to the whole temperature range as the DMI is only present when the chemical potential exceeds the magnetic gap, meaning it exists only in the metallic regime.

Finally, we determine the fluctuation-induced effective Chern-Simons (CS) action,

$$
S_{\text {eff }}^{\mathrm{cs}}=\frac{\sigma}{2} \int d(c t) \int d^{2} r \epsilon^{\mu \nu \lambda} \mathcal{A}_{\mu} \partial_{\nu} \mathcal{A}_{\lambda},
$$

where we have defined the covariant three-potential $\mathcal{A}_{\mu}=$ $\left(\frac{\phi}{c}, \pm \frac{J_{0}}{e v_{\mathrm{F}}} \boldsymbol{d}(\delta \boldsymbol{n})\right)$. The electric potential enters in the time component as usual and the magnetization fluctuations $\delta \boldsymbol{n}$ act as the vector potential $\mathbf{A}$ in the spatial components. Note that the " \pm " applies to the different choices for the vector $\boldsymbol{d}$. The coefficient $\sigma$ arising in Eq. (15) leads to the gap in magnetic susceptibility, in a mechanism closely related to the well-known topologically massive photons in a MaxwellChern-Simons theory [46]. In our case this topological mass is given by

$$
\sigma=\frac{e^{2}}{2 h} \frac{\sinh (\beta m)}{\cosh (\beta \mu)+\cosh (\beta m)} .
$$

We recall here that the topological mass arising in the effective free energy is in general not identical to the Hall conductivity - these quantities differ, for instance, in the metallic regime $[6,29]$, something that is more easily seen in the zero temperature limit. Indeed, for $T=0$ the topological mass and Hall conductivity are given by [6] $\sigma(T=0)=$ $e^{2} \operatorname{sgn}\left(J_{0}\right) H\left(\left|J_{0}\right|-\epsilon_{\mathrm{F}}\right) /(2 h)$ and $\sigma_{x y}(T=0)=e^{2}\left\{\left[\operatorname{sgn}\left(J_{0}\right)-\right.\right.$ $\left.\left.J_{0} / \epsilon_{\mathrm{F}}\right] H\left(\left|J_{0}\right|-\epsilon_{\mathrm{F}}\right)+J_{0} / \epsilon_{\mathrm{F}}\right\} /(2 h)$, respectively. These zero temperature expressions involving the Heaviside step function are identical only when $\epsilon_{\mathrm{F}}<\left|J_{0}\right|$. In fact, $\sigma(T=0)$ vanishes in the metallic regime while $\sigma_{x y}$ is nonzero. This occurs because the Hall conductivity is calculated from the Kubo formula where one first takes the limit $\boldsymbol{q} \rightarrow \mathbf{0}$ and then $\omega \rightarrow 0$, while in case of the topological mass these limits are taken simultaneously. The CS action (15) contains the TME

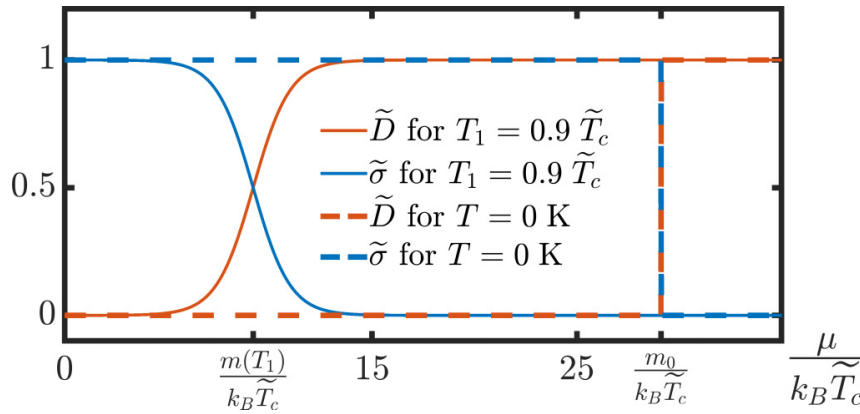

FIG. 3. Crossover of the normalized topological mass $\tilde{\sigma}$ and DMI coupling strength $\tilde{D}$ as a function of the chemical potential at different temperatures. For a vanishing temperature both quantities are step functions. Increasing the temperature causes the step functions to smear out, making coexistence possible. The two quantities always sum up to unity at a given value for the chemical potential.

contribution to the free energy,

$$
F_{\mathrm{TME}}=i \frac{J_{0} \sigma}{e v_{\mathrm{F}}} \int d^{2} r \delta \boldsymbol{n} \cdot[\hat{\mathbf{z}} \times \boldsymbol{d}(-i \nabla) \phi] .
$$

To compare its features to the ones of the DMI we also illustrate its dependency on temperature and chemical potential in Fig. 2(b). Once more it shows that at zero temperature we have a step-function behavior which also approximately extends to finite temperatures. Consequently, the topological mass only exists when the chemical potential lies inside the magnetic gap and is nearly quantized in the bordering regions resulting into plateaus. In comparison to the generated DMI we can see in Fig. 3 that there is a very narrow region where both functions overlap. As a result, the desired simultaneous occurrence of the DMI and the CS action requires fine tuning.

However, upon closer inspection, a different connection between the two terms appears. It turns out that the temperature functions inside both terms complement each other almost perfectly in a temperature and chemical potential plot, as also can be seen for exemplary temperatures in Fig. 3. The two functions are adding up to one creating a plateau that even traverses the chasm that both functions showed individually in the vicinity where the chemical potential crossed the magnetic gap. This means that at the time one of the terms diminishes, the respective other term grows in size equal to the loss of the other, creating a direct correspondence between them. We point out that besides the DMI and CS terms, other interesting terms appear in the effective action, as is shown in explicitly in the SM [43].

Conclusion. We have considered a minimal model for magnetic topological insulators that capture a wealth of interesting properties of the surface of materials such as $\mathrm{MnTe}\left(\mathrm{Bi}_{2} \mathrm{Te}_{3}\right)_{m}$ with $m \geqslant 1[17,19,21,22]$ and $\mathrm{MnSb}_{2} \mathrm{Te}_{4}$ [23,24], and also at the interfaces of the heterostructures $\mathrm{EuS}-\mathrm{Bi}_{2} \mathrm{Se}_{3}$ [12-14]. An important main result of our analysis is the prediction of the survival of the electronic gap on the surface/interface for temperatures above the bulk ordering temperature. In order to provide quantitative results to be compared with experiment, we have combined the effective field theory analysis with DFT and MCS results applied specifically to $\mathrm{MnBi}_{2} \mathrm{Te}_{4}$ and the bilayer system $\mathrm{EuS}-\mathrm{Bi}_{2} \mathrm{Se}_{3}$. We also predict that temperature- 
dependent DMI and TME terms are induced by fluctuations. The latter may give rise to new magnetic phenomena at the surface of magnetic TIs, including the interesting possibility of manipulating skyrmions by external or internal electrical fields.

Acknowledgments. J.v.d.B. acknowledges support by the Deutsche Forschungsgemeinschaft (DFG) through the
Würzburg-Dresden Cluster of Excellence on Complexity and Topology in Quantum Matter - ct.qmat (EXC 2147, project-id 39085490) and through SFB 1143 (project-id 247310070). J.I.F. would like to thank the support of the Alexander von Humboldt Foundation. I.M.E. would like to thank the DFG Priority Program SPP 1666, "Topological Insulators," under Grant No. ER 463/9. The authors thank Ulrike Nitzsche for technical assistance.
[1] C.-X. Liu, S.-C. Zhang, and X.-L. Qi, The quantum anomalous Hall effect: Theory and experiment, Annu. Rev. Condens. Matter Phys. 7, 301 (2016).

[2] T. Yokoyama, J. Zang, and N. Nagaosa, Theoretical study of the dynamics of magnetization on the topological surface, Phys. Rev. B 81, 241410(R) (2010).

[3] I. Garate and M. Franz, Inverse Spin-Galvanic Effect in the Interface between a Topological Insulator and a Ferromagnet, Phys. Rev. Lett. 104, 146802 (2010).

[4] Y. Tserkovnyak and D. Loss, Thin-Film Magnetization Dynamics on the Surface of a Topological Insulator, Phys. Rev. Lett. 108, 187201 (2012).

[5] F. S. Nogueira and I. Eremin, Fluctuation-Induced Magnetization Dynamics and Criticality at the Interface of a Topological Insulator with a Magnetically Ordered Layer, Phys. Rev. Lett. 109, 237203 (2012).

[6] F. S. Nogueira and I. Eremin, Thermal screening at finite chemical potential on a topological surface and its interplay with proximity-induced ferromagnetism, Phys. Rev. B 90, 014431 (2014).

[7] F. S. Nogueira and I. Eremin, Semimetal-insulator transition on the surface of a topological insulator with in-plane magnetization, Phys. Rev. B 88, 085126 (2013).

[8] Y. Tserkovnyak, D. A. Pesin, and D. Loss, Spin and orbital magnetic response on the surface of a topological insulator, Phys. Rev. B 91, 041121(R) (2015).

[9] S. Rex, F. S. Nogueira, and A. Sudbø, Nonlocal topological magnetoelectric effect by Coulomb interaction at a topological insulator-ferromagnet interface, Phys. Rev. B 93, 014404 (2016).

[10] M. Li, W. Cui, J. Yu, Z. Dai, Z. Wang, F. Katmis, W. Guo, and J. Moodera, Magnetic proximity effect and interlayer exchange coupling of ferromagnetic/topological insulator/ferromagnetic trilayer, Phys. Rev. B 91, 014427 (2015).

[11] S. Rex, F. S. Nogueira, and A. Sudbø, Topological magnetic dipolar interaction and nonlocal electric magnetization control in topological insulator heterostructures, Phys. Rev. B 94, 020404(R) (2016).

[12] F. Katmis, V. Lauter, F. S. Nogueira, B. A. Assaf, M. E. Jamer, P. Wei, B. Satpati, J. W. Freeland, I. Eremin, D. Heiman, P. Jarillo-Herrero, and J. S. Moodera, A high-temperature ferromagnetic topological insulating phase by proximity coupling, Nature (London) 533, 513 (2016).

[13] P. Wei, F. Katmis, B. A. Assaf, H. Steinberg, P. Jarillo-Herrero, D. Heiman, and J. S. Moodera, Exchange-Coupling-Induced Symmetry Breaking in Topological Insulators, Phys. Rev. Lett. 110, 186807 (2013).

[14] Q. I. Yang, M. Dolev, L. Zhang, J. Zhao, A. D. Fried, E. Schemm, M. Liu, A. Palevski, A. F. Marshall, S. H. Risbud, and A. Kapitulnik, Emerging weak localization effects on a topological insulator-insulating ferromagnet $\left(\mathrm{Bi}_{2} \mathrm{Se}_{3}-\mathrm{EuS}\right)$ interface, Phys. Rev. B 88, 081407(R) (2013).

[15] C. Lee, F. Katmis, P. Jarillo-Herrero, J. S. Moodera, and N. Gedik, Direct measurement of proximity-induced magnetism at the interface between a topological insulator and a ferromagnet, Nat. Commun. 7, 12014 (2016).

[16] Q. L. He, X. Kou, A. J. Grutter, G. Yin, L. Pan, X. Che, Y. Liu, T. Nie, B. Zhang, S. M. Disseler, B. J. Kirby, W. Ratcliff, II, Q. Shao, K. Murata, X. Zhu, G. Yu, Y. Fan, M. Montazeri, X. Han, J. A. Borchers, and K. L. Wang, Tailoring exchange couplings in magnetic topological-insulator/antiferromagnet heterostructures, Nat. Mater. 16, 94 (2017).

[17] M. M. Otrokov, I. I. Klimovskikh, H. Bentmann, D. Estyunin, A. Zeugner, Z. S. Aliev, S. Gaß, A. U. B. Wolter, A. V. Koroleva, A. M. Shikin, M. Blanco-Rey, M. Hoffmann, I. P. Rusinov, A. Yu. Vyazovskaya, S. V. Eremeev, Yu. M. Koroteev, V. M. Kuznetsov, F. Freyse, J. Sánchez-Barriga, I. R. Amiraslanov et al., Prediction and observation of an antiferromagnetic topological insulator, Nature (London) 576, 416 (2019).

[18] Y. Gong, J. Guo, J. Li, K. Zhu, M. Liao, X. Liu, Q. Zhang, L. Gu, L. Tang, X. Feng, D. Zhang, W. Li, C. Song, L. Wang, P. Yu, X. Chen, Y. Wang, H. Yao, W. Duan, Y. Xu et al., Experimental realization of an intrinsic magnetic topological insulator, Chin. Phys. Lett. 36, 076801 (2019).

[19] A. Zeugner, F. Nietschke, A. U. B. Wolter, S. Gaß, R. C. Vidal, T. R. F. Peixoto, D. Pohl, C. Damm, A. Lubk, R. Hentrich, S. K. Moser, C. Fornari, C. H. Min, S. Schatz, K. Kißner, M. Ünzelmann, M. Kaiser, F. Scaravaggi, B. Rellinghaus, K. Nielsch et al., Chemical aspects of the candidate antiferromagnetic topological insulator $\mathrm{MnBi}_{2} \mathrm{Te}_{4}$, Chem. Mater. 31, 2795 (2019).

[20] R. C. Vidal, H. Bentmann, T. R. F. Peixoto, A. Zeugner, S. Moser, C.-H. Min, S. Schatz, K. Kißner, M. Ünzelmann, C. I. Fornari, H. B. Vasili, M. Valvidares, K. Sakamoto, D. Mondal, J. Fujii, I. Vobornik, S. Jung, C. Cacho, T. K. Kim, R. J. Koch, C. Jozwiak, A. Bostwick, J. D. Denlinger, E. Rotenberg, J. Buck, M. Hoesch, F. Diekmann, S. Rohlf, M. Kalläne, K. Rossnagel, M. M. Otrokov, E. V. Chulkov, M. Ruck, A. Isaeva, and F. Reinert, Surface states and Rashba-type spin polarization in antiferromagnetic $\mathrm{MnBi}_{2} \mathrm{Te}_{4}(0001)$, Phys. Rev. B 100, 121104(R) (2019)

[21] M. M. Otrokov, I. P. Rusinov, M. Blanco-Rey, M. Hoffmann, A. Yu. Vyazovskaya, S. V. Eremeev, A. Ernst, P. M. Echenique, A. Arnau, and E. V. Chulkov, Unique ThicknessDependent Properties of the van der Waals Interlayer Antiferromagnet $\mathrm{MnBi}_{2} \mathrm{Te}_{4}$ Films, Phys. Rev. Lett. 122, 107202 (2019).

[22] R. C. Vidal, A. Zeugner, J. I. Facio, R. Ray, M. H. Haghighi, A. U. B. Wolter, L. T. Corredor Bohorquez, F. Caglieris, 
S. Moser, T. Figgemeier, T. R. F. Peixoto, H. B. Vasili, M. Valvidares, S. Jung, C. Cacho, A. Alfonsov, K. Mehlawat, V. Kataev, C. Hess, M. Richter, B. Büchner, J. van den Brink, M. Ruck, F. Reinert, H. Bentmann, and A. Isaeva, Topological Electronic Structure and Intrinsic Magnetization in $\mathrm{MnBi}_{4} \mathrm{Te}_{7}$ : $\mathrm{A} \mathrm{Bi}_{2} \mathrm{Te}_{3}$ Derivative with a Periodic Mn Sublattice, Phys. Rev. X 9, 041065 (2019).

[23] B. Chen, F. Fei, D. Zhang, B. Zhang, W. Liu, S. Zhang, P. Wang, B. Wei, Y. Zhang, Z. Zuo et al., Intrinsic magnetic topological insulator phases in the $\mathrm{Sb}$ doped $\mathrm{MnBi}_{2} \mathrm{Te}_{4}$ bulks and thin flakes, Nat. Commun. 10, 4469 (2019).

[24] S. Wimmer, J. Sánchez-Barriga, P. Küppers, A. Ney, E. Schierle, F. Freyse, O. Caha, J. Michalicka, M. Liebmann, D. Primetzhofer, M. Hoffmann, A. Ernst, M. M. Otrokov, G. Bihlmayer, E. Weschke, B. Lake, E. V. Chulkov, M. Morgenstern, G. Bauer, G. Springholz, and O. Rader, Ferromagnetic $\mathrm{MnSb}_{2} \mathrm{Te}_{4}$ : A topological insulator with magnetic gap closing at high Curie temperatures of 45-50 K, arXiv:2011.07052.

[25] T. Hirahara, S. V. Eremeev, T. Shirasawa, Y. Okuyama, T. Kubo, R. Nakanishi, R. Akiyama, A. Takayama, T. Hajiri, S.-i. Ideta, M. Matsunami, K. Sumida, K. Miyamoto, Y. Takagi, K. Tanaka, T. Okuda, T. Yokoyama, S.-i. Kimura, S. Hasegawa, and E. V. Chulkov, Large-gap magnetic topological heterostructure formed by subsurface incorporation of a ferromagnetic layer, Nano Lett. 17, 3493 (2017).

[26] T. Hirahara, M. M. Otrokov, T. T. Sasaki, K. Sumida, Y. Tomohiro, S. Kusaka, Y. Okuyama, S. Ichinokura, M. Kobayashi, Y. Takeda, K. Amemiya, T. Shirasawa, S. Ideta, K. Miyamoto, K. Tanaka, S. Kuroda, T. Okuda, K. Hono, S. V. Eremeev, and E. V. Chulkov, Fabrication of a novel magnetic topological heterostructure and temperature evolution of its massive Dirac cone, Nat. Commun. 11, 4821 (2020).

[27] X.-L. Qi, T. L. Hughes, and S.-C. Zhang, Topological field theory of time-reversal invariant insulators, Phys. Rev. B 78, 195424 (2008).

[28] S. Ryu, J. E. Moore, and A. W. W. Ludwig, Electromagnetic and gravitational responses and anomalies in topological insulators and superconductors, Phys. Rev. B 85, 045104 (2012).

[29] C. Tutschku, F. S. Nogueira, C. Northe, J. van den Brink, and E. M. Hankiewicz, Temperature and chemical potential dependence of the parity anomaly in quantum anomalous Hall insulators, Phys. Rev. B 102, 205407 (2020).

[30] F. S. Nogueira, I. Eremin, F. Katmis, J. S. Moodera, J. van den Brink, and V. P. Kravchuk, Fluctuation-induced Néel and Bloch skyrmions at topological insulator surfaces, Phys. Rev. B 98, 060401(R) (2018).

[31] K. L. Tiwari, J. Lavoie, T. Pereg-Barnea, and W. A. Coish, Tunable skyrmion-skyrmion binding on the surface of a topological insulator, Phys. Rev. B 100, 125414 (2019).

[32] S. Zhang, F. Kronast, G. van der Laan, and T. Hesjedal, Realspace observation of skyrmionium in a ferromagnet-magnetic topological insulator heterostructure, Nano Lett. 18, 1057 (2018).

[33] J. Kim, K.-W. Kim, H. Wang, J. Sinova, and R. Wu, Understanding the Giant Enhancement of Exchange Interaction in $\mathrm{Bi}_{2} \mathrm{Se}_{3}-\mathrm{EuS}$ Heterostructures, Phys. Rev. Lett. 119, 027201 (2017).

[34] H. Wang, Y. Liu, P. Wu, W. Hou, Y. Jiang, X. Li, C. Pandey, D. Chen, Q. Yang, H. Wang, D. Wei, N. Lei, W. Kang, L. Wen,
T. Nie, W. Zhao, and K. L. Wang, Above room-temperature ferromagnetism in wafer-scale two-dimensional van der Waals $\mathrm{Fe}_{3} \mathrm{GeTe}_{2}$ tailored by a topological insulator, ACS Nano 14, 10045 (2020).

[35] J. A. Krieger, Y. Ou, M. Caputo, A. Chikina, M. Döbeli, M.-A Husanu, I. Keren, T. Prokscha, A. Suter, C.-Z. Chang, J. S. Moodera, V. N. Strocov, and Z. Salman, Do topology and ferromagnetism cooperate at the $\mathrm{EuS} / \mathrm{Bi}_{2} \mathrm{Se}_{3}$ interface? Phys. Rev. B 99, 064423 (2019).

[36] A. I. Figueroa, F. Bonell, M. G. Cuxart, M. Valvidares, P. Gargiani, G. van der Laan, A. Mugarza, and S. O. Valenzuela, Absence of Magnetic Proximity Effect at the Interface of $\mathrm{Bi}_{2} \mathrm{Se}_{3}$ and $(\mathrm{Bi}, \mathrm{Sb})_{2} \mathrm{Te}_{3}$ with EuS, Phys. Rev. Lett. 125, 226801 (2020).

[37] J. Wu, F. Liu, M. Sasase, K. Ienaga, Y. Obata, R. Yukawa, K. Horiba, H. Kumigashira, S. Okuma, T. Inoshita et al., Natural van der Waals heterostructural single crystals with both magnetic and topological properties, Sci. Adv. 5, eaax9989 (2019).

[38] J. W. Negele and H. Orland, Quantum Many-Particle Systems (Addison-Wesley, Reading, MA, 1988).

[39] S. Sachdev, Quantum Phase Transitions, 2nd ed. (Cambridge University Press, Cambridge, UK, 2011).

[40] S. V. Eremeev, V. N. Men'shov, V. V. Tugushev, and E. V. Chulkov, Interface induced states at the boundary between a $3 \mathrm{D}$ topological insulator $\mathrm{Bi}_{2} \mathrm{Se}_{3}$ and a ferromagnetic insulator $\mathrm{EuS}$, J. Magn. Magn. Mater. 383, 30 (2015).

[41] H. Zhang, C.-X. Liu, X.-L. Qi, X. Dai, Z. Fang, and S.-C. Zhang, Topological insulators in $\mathrm{Bi}_{2} \mathrm{Se}_{3}, \mathrm{Bi}_{2} \mathrm{Te}_{3}$ and $\mathrm{Sb}_{2} \mathrm{Te}_{3}$ with a single Dirac cone on the surface, Nat. Phys. 5, 438 (2009).

[42] A. Tan, V. Labracherie, N. Kunchur, A. U. B. Wolter, J. Cornejo, J. Dufouleur, B. Büchner, A. Isaeva, and R. Giraud, Metamagnetism of Weakly Coupled Antiferromagnetic Topological Insulators, Phys. Rev. Lett. 124, 197201 (2020).

[43] See Supplemental Material at http://link.aps.org/supplemental/ 10.1103/PhysRevResearch.3.L032014 for details on the analytic calculations, density functional theory results, and Monte Carlo simulations, which includes Refs. [47-55].

[44] B. Li, J.-Q. Yan, D. M. Pajerowski, E. Gordon, A.-M. Nedić, Y. Sizyuk, L. Ke, P. P. Orth, D. Vaknin, and R. J. McQueeney, Competing Magnetic Interactions in the Antiferromagnetic Topological Insulator $\mathrm{MnBi}_{2} \mathrm{Te}_{4}$, Phys. Rev. Lett. 124, 167204 (2020).

[45] A. Mauger and C. Godart, The magnetic, optical, and transport properties of representatives of a class of magnetic semiconductors: The europium chalcogenides, Phys. Rep. 141, 51 (1986).

[46] S. Deser, R. Jackiw, and S. Templeton, Three-Dimensional Massive Gauge Theories, Phys. Rev. Lett. 48, 975 (1982).

[47] J. P. Perdew, K. Burke, and M. Ernzerhof, Generalized Gradient Approximation Made Simple, Phys. Rev. Lett. 77, 3865 (1996).

[48] K. Koepernik and H. Eschrig, Full-potential nonorthogonal local-orbital minimum-basis band-structure scheme, Phys. Rev. B 59, 1743 (1999).

[49] https://www.fplo.de.

[50] R. C. Vidal, H. Bentmann, J. I. Facio, T. Heider, P. Kagerer, C. I. Fornari, T. R. F. Peixoto, T. Figgemeier, S. Jung, C. Cacho, B. Büchner, J. van den Brink, C. M. Schneider, L. Plucinski, E. F. Schwier, K. Shimada, M. Richter, A. Isaeva, and F. Reinert, Orbital Complexity in Intrinsic Magnetic Topological 
Insulators $\mathrm{MnBi}_{4} \mathrm{Te}_{7}$ and $\mathrm{MnBi}_{6} \mathrm{Te}_{10}$, Phys. Rev. Lett. 126, 176403 (2021).

[51] Y. J. Chen, L. X. Xu, J. H. Li, Y. W. Li, H. Y. Wang, C. F. Zhang, H. Li, Y. Wu, A. J. Liang, C. Chen, S. W. Jung, C. Cacho, Y. H. Mao, S. Liu, M. X. Wang, Y. F. Guo, Y. Xu, Z. K. Liu, L. X. Yang, and Y. L. Chen, Topological Electronic Structure and its Temperature Evolution in Antiferromagnetic Topological Insulator $\mathrm{MnBi}_{2} \mathrm{Te}_{4}$, Phys. Rev. X 9, 041040 (2019).

[52] C. Pérez Vicente, J. L. Tirado, K. Adouby, J. C. Jumas, A. Abba Touré, and G. Kra, X-ray diffraction and ${ }^{119} \mathrm{Sn}$ Mössbauer spectroscopy study of a new phase in the $\mathrm{Bi}_{2} \mathrm{Se}_{3}-\mathrm{SnSe}$ system: $\mathrm{SnBi}_{4} \mathrm{Se}_{7}$, Inorg. Chem. 38, 2131 (1999).
[53] P. Wachter, The optical electrical and magnetic properties of the europium chalcogenides and the rare earth pnictides, CRC Crit. Rev. Solid State Sci. 3, 189 (1972).

[54] T. Story, C. H. W. Swüste, H. J. M. Swagten, W. J. M. de Jonge, A. Stachow-Wójcik, A. Twardowski, M. Arciszewska, W. Dobrowolski, R. R. Gałazka, and A. Yu. Sipatov, Magnetic anisotropy in Eus-PbS multilayers, Acta Phys. Pol., A 97, 435 (2000).

[55] M. Ghaemi, G. A. Parsafar, and M. Ashrafizaadeh, Calculation of the critical temperature for 2- and 3-dimensional Ising models and for 2-dimensional Potts models using the transfer matrix method, J. Phys. Chem. B 105, 10355 (2001). 